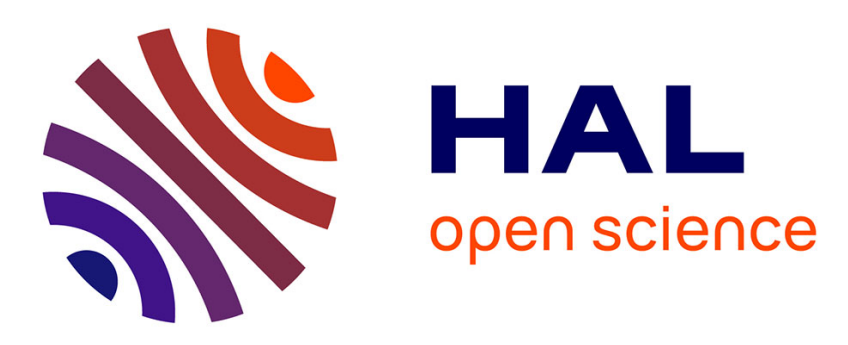

\title{
A hybrid stimulation strategy for suppression of spiral waves in cardiac tissue
}

Binbin Xu, Sabir Jacquir, Gabriel Laurent, Jean-Marie Bilbault, Stéphane Binczak

\section{- To cite this version:}

Binbin Xu, Sabir Jacquir, Gabriel Laurent, Jean-Marie Bilbault, Stéphane Binczak. A hybrid stimulation strategy for suppression of spiral waves in cardiac tissue. Chaos, Solitons \& Fractals, 2011, 44 (8), pp.633-639. 10.1016/j.chaos.2011.05.014 . hal-00631743

\section{HAL Id: hal-00631743 https://u-bourgogne.hal.science/hal-00631743}

Submitted on 13 Oct 2011

HAL is a multi-disciplinary open access archive for the deposit and dissemination of scientific research documents, whether they are published or not. The documents may come from teaching and research institutions in France or abroad, or from public or private research centers.
L'archive ouverte pluridisciplinaire HAL, est destinée au dépôt et à la diffusion de documents scientifiques de niveau recherche, publiés ou non, émanant des établissements d'enseignement et de recherche français ou étrangers, des laboratoires publics ou privés. 


\section{- A Hybrid Stimulation Strategy for Suppression of Spiral \\ Waves in Cardiac Tissue}

\author{
Binbin Xu, Sabir Jacquir, Gabriel Laurent, Jean-Marie Bilbault, \\ Stéphane Binczak \\ CNRS UMR 5158 Dijon France \\ Université de Bourgogne, LE2I Dijon France
}

\section{Abstract}

$8 \quad$ Atrial fibrillation (AF) is the most common cardiac arrhythmia whose me-

- chanisms are thought to be mainly due to the self perpetuation of spiral waves

10 (SW). To date, available treatment strategies (antiarrhythmic drugs, radiofre-

11 quency ablation of the substrate, electrical cardioversion) to restore and to

12 maintain a normal sinus rhythm have limitations and are associated with AF

13 recurrences. The aim of this study was to assess a way of suppressing SW by

$\mathbf{1 4}$ applying multifocal electrical stimulations in a simulated cardiac tissue using

15 a 2D FitzHugh-Nagumo model specially convenient for AF investigations. We

16 identified stimulation parameters for successful termination of SW. However,

17 SW reinduction, following the electrical stimuli, leads us to develop a hybrid

18 strategy based on sodium channel modification for the simulated tissue.

19 Version : 175

2о 15 avril $2011,11: 18: 50$

Email addresses: sjacquir@u-bourgogne.fr (Sabir Jacquir), stbinc@u-bourgogne.fr ( StÃl'phane Binczak)

Preprint submitted to Elsevier

15 avril 2011 


\section{1. Introduction}

$2 \quad$ Although human data support the idea that rotors are a crucial mechanism 3 for fibrillation maintenance in both atria and ventricles, there are clear diffe-

4 rences between the 2 chamber types. Ventricular fibrillation (VF), responsible for 5 sudden cardiac death, can be considered as a lethal "three-dimensional" cardiac - arrhythmia with multiple micro re-entries located deep inside the endocardial 7 muscle [1]. VF has been widely studied [2, 3, 4], but because of electrophy8 siological characteristics, the only treatment available is indeed an electrical - defibrillation via automated external defibrillators (AED), or via implantable 10 cardiac defibrillators (ICD). Conversely, most of "two-dimensional" macro re11 entry ventricular tachycardia (VT) can be stopped by rapid burst of stimulations 12 delivered by an ICD via a single right ventricular lead [5].

13 As opposed to VF, atrial fibrillation (AF) is associated with a better outcome 14 but it is by far the most frequently diagnosed cardiac arrhythmia affecting, 15 for example, approximately $1 \%$ of the adult population of the United States.

16 Recent clinical trial evidence suggests that the presence of AF is an independent

17 predictor of morbidity and mortality. One of the primary goals of paroxysmal

18 and persistent AF treatment is to relieve symptoms by a rhythm control strategy

$19 \quad[6]$.

2o Endocardial walls of the atria are much thinner than their ventricular coun21 terparts, therefore AF may be considered as a "two-dimensional" cardiac ar22 rhythmia. Specifically, therapy for restoring and maintaining a normal sinus 23 rhythm is multidimensional with treatment options spanning from pharmacolo24 gic therapy to electrical cardioversion and catheter-based radiofrequency abla25 tion [7]. But even combined, these methods have limited efficacy as the AF re26 currence rate is high, and are limited by numerous side effects [8]. To date, there 27 are two known concepts to restore a normal sinus rhythm by using electrical sti28 mulation. The first one is to "reset" the whole heart by applying high voltage via a dedicated defibrillation device (internal or external) in order to extinguish most of the fibrillatory wavefronts. External cardiac defibrillation cannot be rea- 
1 lized very often as it has to be done under general anesthesia. Maintenance of 2 normal sinus rhythm using repeated internal cardioversion shocks via implan3 ted devices has also been shown to be effective and safe, however increase in 4 defibrillation thresholds and patient intolerance due to the pain were the main 5 reasons for discontinuation of this therapy [9]. The second way of suppressing - AF is based on Allessie's concept asserting that there is a critical number of 7 micro-reentries below which the cardiac arrhythmia can self terminate [10].

$8 \quad$ Micro-reentries can be observed experimentally or numerically as spiral waves 9 (SW). The rotors of SW, defined as rotating motors that give rise to the spiraling wavefronts, gyrate at relatively high frequencies [11]. From such rotors, SW propagate throughout the myocardium in very complex ways [3], for example SW meandering and drifting, whose dynamics have been well studied $[12,13,14]$. It is known that, under certain conditions, electrical stimuli can modify SW dynamics $[15,16,17]$. Moreover, they can terminate SW either by preventing rotor formation and maintenance, by re-driving / pacing SW [18] or by feedback control [19]. This hypothesis could explain the global mechanism of defibrillation by electrical stimulation. Namely, at cellular level, the propagation of action potential in excitable medium is controlled by voltage-gated ion channels in cells. These channels are shut when the membrane potential is near the resting potential. They rapidly begin to open if the membrane potential increases to the threshold value. However, as opposed to VT, AF can't be stopped by single site burst stimulations via a pacing lead [7], but a multifocal and simultaneous stimulation may help to extinguish most or all fibrillatory wavefronts in a selected area by resetting cellular membrane potential under its threshold value.

This approach has already been investigated in previous studies. Allessie has shown that, during electrically induced AF in chronically instrumented conscious dogs, it was possible to control the local fibrillatory process by rapid focal pacing through a grid of electrodes at the left atrial appendage [20]. However, this method failed to terminate AF as the area under control was very limited. Other authors have used an array of control points in order to terminate spatiotemporal chaos in excitable media and focused mainly on the 
1 stimulation amplitude [21]. However, we strongly believe that there are many

2 other parameters to be taken into account.

$3 \quad$ Other methods to suppress SW based on boundaries conditions or spatial

4 inhomogeneity $[22,23]$ are either difficult in controlling the inhomogeneity of a

5 real cardiac tissue, or are associated with the generation of new waves. In [24],

- a spatially extended mesh which could be more successful in the elimination

7 of spiral turbulence has been mentioned. But the optimal parameters of the

8 controlling signals have not been defined. Moreover, these techniques have strong

- limitations as they not only eliminate SW, but also reinduce new ones.

In fact, the ideal atrial stimulation/defibrillation method should suppress enough SW in a dedicated region for the whole heart to stop fibrillating, but without generating new ones.

In order to address this major concern, we studied two different options by using $2 D$ numerical simulations of the model, as described in section 2. Then, we optimized the stimulation parameters (section 3). For example, we defined the number of required stimulated sites via an electrode array; we optimized the frequency and duty cycle of the stimulation, and the output amplitude which has to be set as low as possible to prevent from new SW and to protect the whole heart from arrhythmia re-introduction. But this minimal amplitude being still too large, we investigated then a second option, in developing a hybrid strategy based on a sodium channel modification of the simulated tissue. By increasing the threshold of new SW generation, we showed that it is possible to protect the tissue from arrhythmia re-induction. We sought to identify the best balance between cells number needed to be stimulated compared to the number of total cells simulated and the SW generation threshold. In the last section 4, we concluded this paper and gave some future prospects of this work.

\section{Model and methods}

In literature, there are many cardiac excitation models, for example, LuoRudy 1 and 2 models [25, 26], Ten Tusscher-Noble-Panfilov model [27]. These 
1 models represent an interesting way to investigate the heart activity at cellu2 lar level, but the control of their parameters is quite complex. The FitzHugh3 Nagumo (FHN) model [28, 29] remains simple and keeps the essential mathe4 matical properties of excitation and propagation from the electrochemical pro5 perties of sodium and potassium ion flows. FHN model is then used to study - the ionic current of nerve or cardiac membrane [30, 31], completed by adding 7 terms to a non-linear "relaxation oscillators" dimensionless equation. We start s from the generic ionic equation of excitable medium with realistic units,

$$
C_{m} \frac{\partial V}{\partial t}=C_{m} d \Delta V(x, y, t)-i_{N a}-i_{\text {other }}+i_{s t i m}
$$

9 where $C_{m}$ is the membrane capacitance ; $V(x, y, t)$ is the transmembrane poten10 tial at location $(x, y)$ at time $t ; d$ is the diffusion parameter; $\Delta$ is the continuous 11 Laplace operator; $i_{N a}$ is the sodium current; $i_{\text {other }}$ is the sum of other ionic 12 currents and $i_{\text {stim }}$ is the stimulus current.

The dynamics of the currents in FHN model are characterized as Fast-Slow ones, where the sodium one is the fast part and is expressed as

$$
i_{N a}=k \cdot g_{N a} \cdot V\left(V-V_{a}\right)\left(V-V_{b}\right) .
$$

15 Biologically, $k$ intervenes in the control of the sodium current dynamics and 16 kinetics of sodium channels. In this model, changing $k$ results in modifying

17 the dynamics of sodium current, then the value and the position of nullclines

18 in the phase space. $g_{N a}$ is the maximum sodium conductance; $V_{a}$ and $V_{b}$ are 19 respectively the activation and inactivation potential;

2o Substituting Eq.(2) into Eq.(1), we obtain :

$$
C_{m} \frac{\partial V}{\partial t}=C_{m} d \Delta V-k \cdot g_{N a} \cdot V\left(V-V_{a}\right)\left(V-V_{b}\right)-i_{\text {other }}+i_{s t i m}
$$

${ }_{21}$ Setting that $v=\frac{V}{V_{b}}, \alpha=\frac{V_{a}}{V_{b}}$ and converting the continuous Laplace operator 22 to discrete Laplace operator with spatial step $\delta h$, yields :

$$
\frac{\partial v}{\partial \tau}=D \Delta v-k \cdot g_{N a} \cdot v(v-\alpha)(v-1)-I_{o t h e r}+I_{s t i m},
$$


with :

$$
\begin{aligned}
\tau & =\frac{g_{N a} \cdot V_{b}^{2}}{C_{m}} \cdot t \\
D & =C_{m} \frac{1}{g_{N a} \cdot V_{b}^{2} \cdot \delta h^{2}} \cdot d \\
I_{s t i m} & =\frac{1}{g_{N a} \cdot V_{b}^{3}} \cdot i_{s t i m}
\end{aligned}
$$

1 where $I_{\text {stim }}$, the normalized stimulus current, is defined by :

$$
\left\{\begin{aligned}
I_{\text {stim }}(x, y, \tau) & =A(x, y) \text { rect }\left(\frac{F}{\theta} \tau\right) * \sum_{n=0}^{N} \delta\left(\tau-\frac{n}{F}\right) \\
A(x, y) & = \begin{cases}A, & \text { if cell }(x, y) \text { is stimulated } \\
0, & \text { otherwise }\end{cases}
\end{aligned}\right.
$$

= $A(x, y) \cdot \operatorname{rect}\left(\frac{F}{\theta} \tau\right)$ is a rectangular pulse with amplitude $A(x, y)$, frequency $F$, 3 duty cycle $\theta$, duration $\frac{\theta}{F}$, and centered in time $\tau=0$. The function $\sum_{n=0}^{N} \delta\left(t-\frac{n}{F}\right)$ 4 is a Dirac comb corresponding to a series of Dirac delta functions spaced at 5 intervals of $\frac{n}{F}$.

- The current $I_{\text {other }}$ in FHN model is introduced by a recovery variable $w$ 7 which indicates the capacity of the medium to revert to its resting state after 8 the propagation of impulsions :

$$
\frac{\partial w}{\partial \tau}=\varepsilon(v-\gamma w)
$$

9 where $\varepsilon$ and $\gamma$ are dimensionless constant parameters.

10 The above equations allow us to obtain the realistic values from numerical 11 ones and vice versa. Some typical values for the realistic parameters are used 12 here : $C_{m}=10^{-6} \mathrm{~F} \mathrm{~cm}^{-2}, g_{N a}=23 \mathrm{mS} \mathrm{V}^{-2} \mathrm{~cm}^{-2}, V_{b}=122 \mathrm{mV}([30,32])$, $13 d=0.026 \mathrm{~cm}^{2} \mathrm{~s}^{-1}$. However, considering the difference of action potentials (AP) ${ }_{14}$ lengths ${ }^{1}$ and the different properties of cell models, this conversion can only 15 provide some qualitative features.

1. Sodium-based AP lasts for less than $1 \mathrm{~ms}$, calcium-based AP lasts for $100 \mathrm{~ms}$ or longer and normal human atria1 AP lasts usually for $383 \pm 103 \mathrm{~ms}$ [33]. 
1

2 sic stimuli from external electrodes distributed periodically in the system. The

3 cardiac tissue is covered by an electrodes network. The size of each electrode is

4 set to be the same as a cardiac cell's. The electrodes network has a square grid

5 form but other patterns and sizes could be considered.

\section{Optimization of parameters and Results}

7 Simulations were performed with an isotropic cardiac tissue of $200 \times 200$ cells

$8 \operatorname{grid}(\delta h=280 \mu \mathrm{m}, \varepsilon=0.005, \gamma=0$ and $\alpha=0.1)$ and implanted with a Runge-

9 Kutta scheme including Neumann boundary conditions. For the readability of

10

11 our results, we call Stimulation Rate (SR) the ratio between the number of cells under stimulation and the total cell number in the cardiac tissue. $\mathrm{SR}=100 \%$ corresponds to the case where all cardiac cells are stimulated. An illustration of the suppression process of SW corresponding to a case $\mathrm{SR}=2.1 \%$, is shown in Fig. 1 with the parameter $k=1$.

Three categories of simulations have been carried out to optimize the suppression of SW : global stimulation, wave generation threshold by stimulation and hybrid stimulation.

\subsection{Global stimulation}

In the global stimulation case, the effects of the parameters of the stimulation signal on the suppression of SW are investigated. Under the hypothesis that there are SW evolving on the tissue, the whole tissue is stimulated. The results are presented by the relationship between the SR and $I_{\text {stim }}$ parameters (amplitude, frequency and duty cycle), as shown in Figs. 2, 3 and 4.

There is a decreasing relationship between SR and each of these stimulation parameters. Indeed, the larger the applied energy is, the smaller the number of stimulated cells can be. However, there exists asymptotically a minimal stimulated cells number needed in the SW suppression process, whatever are the amplitude, frequency or duty cycle of the stimulus, which is in agreement with

[20]. The slope is very important for smaller amplitudes, lower frequencies and 
1 smaller duty cycles. This means that in these regions, small variations of ampli-

2 tude or frequency could greatly change the result of suppression process of SW.

3 Furthermore, decreasing too much both parameters would increase SR which

4 is contradictory to our main goal : stimulating less cells. As a consequence,

5 a reasonable compromise should be taken. In following simulations, we take

- $A=83.53 \mathrm{\mu} \mathrm{A} \mathrm{cm}^{-2}, F=21.40 \mathrm{~Hz}, \theta=50 \%$ as our benchmark parameters.

7 Observing these results, it seems possible to suppress SW by choosing the

8 appropriate parameters, but this is valuable only if the stimulation grid covers

9 the whole tissue. This global stimulation is not possible in the clinical case, because it is difficult to stimulate the whole cardiac tissue. If this grid covers

11 just a localized area of the tissue, SW can actually be suppressed in this area,

12 but outside this area the stimulation provokes other waves (see wave fronts in

13 Fig. 5c and 5d, propagating with a growing rough square shape outwards the

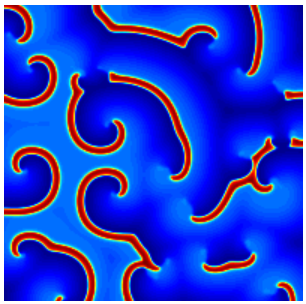

(a)

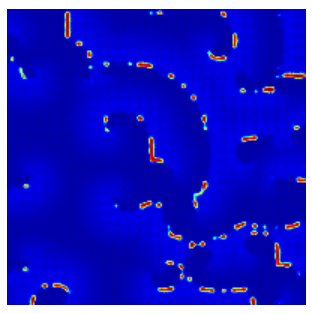

(d)

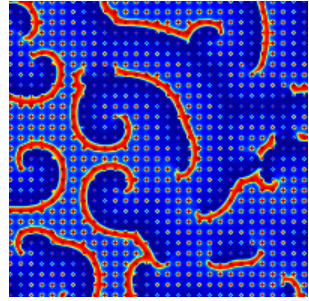

(b)

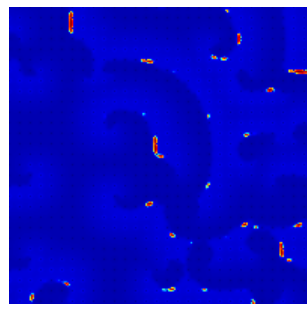

(e)

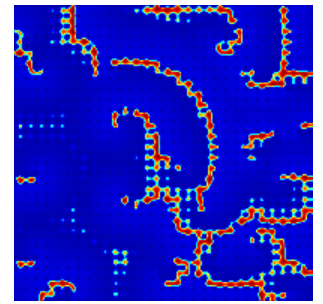

(c)

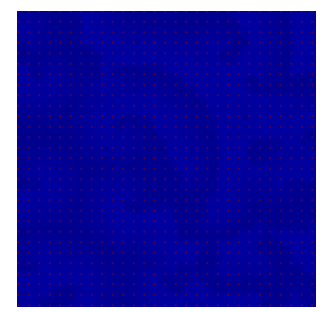

(f)

Figure 1: Suppression of SW at different times : (a) $t=0 \mathrm{~ms}$; (b) $t=26.29 \mathrm{~ms}$; (c) $t=181.11 \mathrm{~ms}$; (d) $t=219.09 \mathrm{~ms}$; (e) $t=265.82 \mathrm{~ms}$; (f) $t=391.43 \mathrm{~ms}$. (Tissue size $200 \times 200$ cells grid, $k=1, A=375.88 \mu \mathrm{A} \mathrm{cm}^{-2}, F=21.40 \mathrm{~Hz}$, $\theta=50 \%, \mathrm{SR}=2.1 \%$ ). 


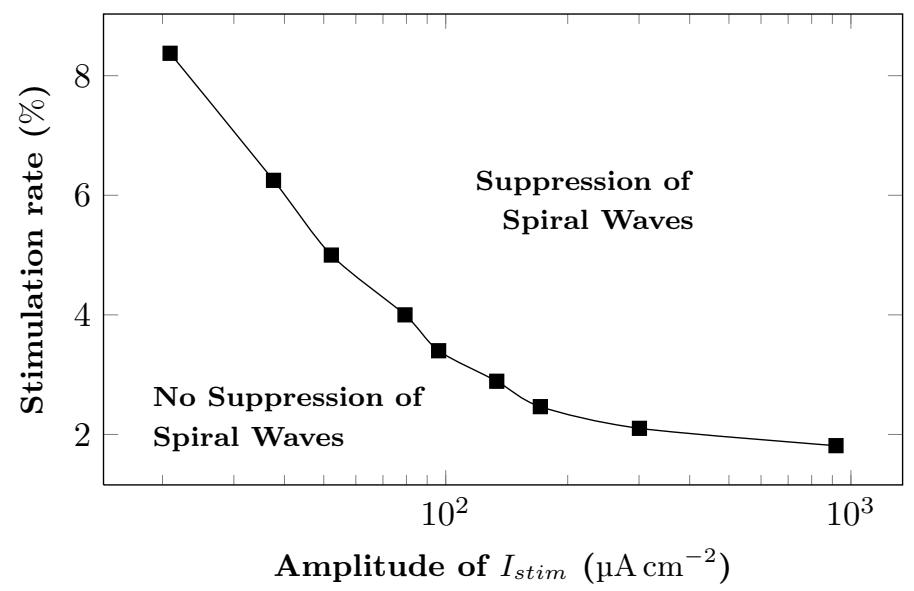

Figure 2: SR vs. amplitude of stimulus $(F=21.40 \mathrm{~Hz}, \theta=50 \%, k=1)$.

1 stimulated area). In certain conditions, for instance : with local inhomogeneity,

2 presence of obstacles or collision of waves, the new generated wave could be 3 transformed into spiral waves. This implies that the amplitude of $I_{\text {stim }}$ is above 4 the threshold of new wave generation. Consequently this kind of stimulation 5 cannot be considered as a real suppression of SW. As a result, it is necessary to 6 determine this threshold as described in the following subsection.

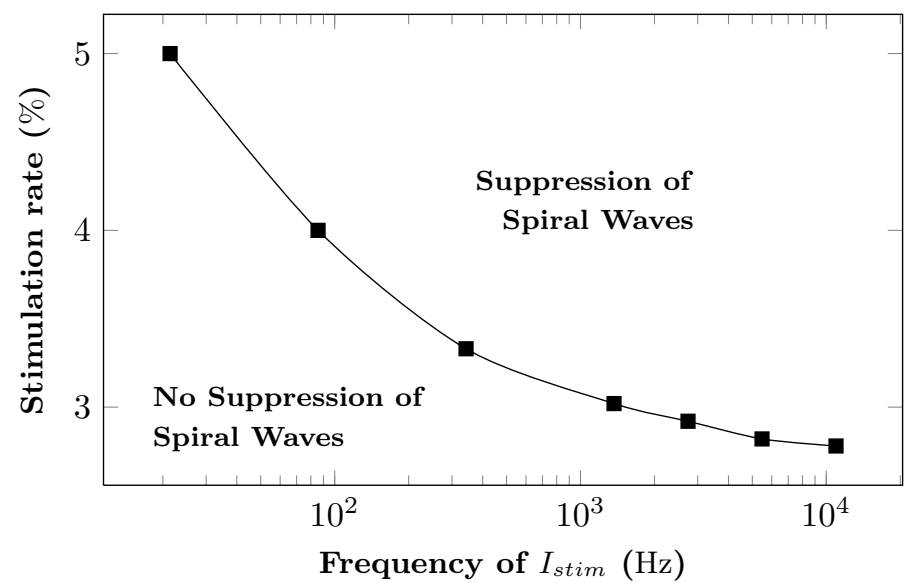

FigURE 3: SR vs. frequency of stimulus $\left(A=83.53 \mu \mathrm{Acm}^{-2}, \theta=50 \%, k=1\right)$. 


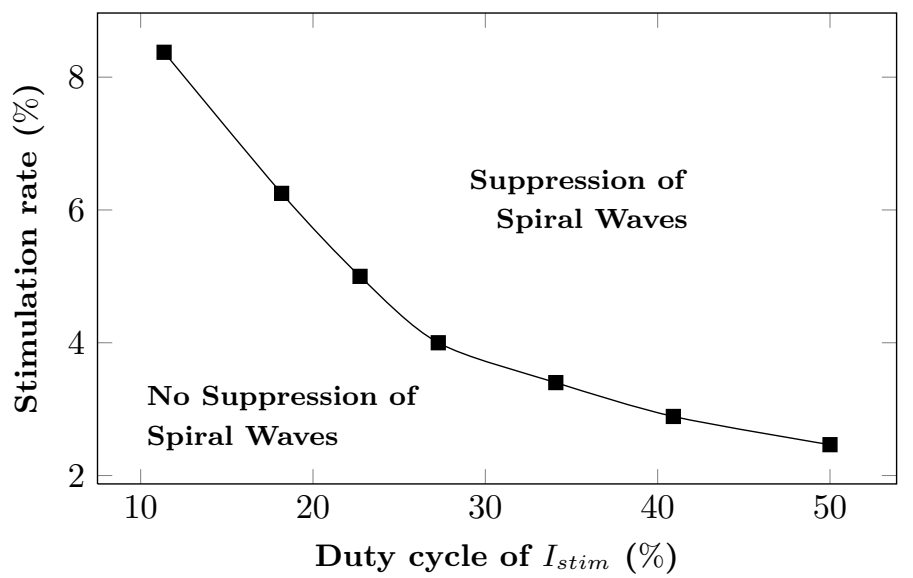

Figure 4: SR vs. duty cycle of stimulus $\left(A=83.53 \mu \mathrm{A} \mathrm{cm}{ }^{-2}, F=21.40 \mathrm{~Hz}\right.$, $k=1)$.

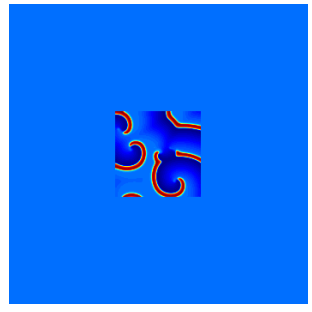

(a)

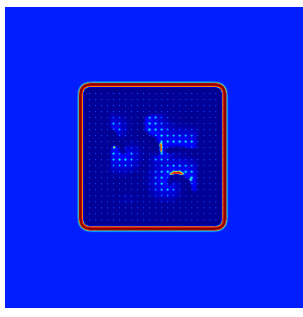

(c)

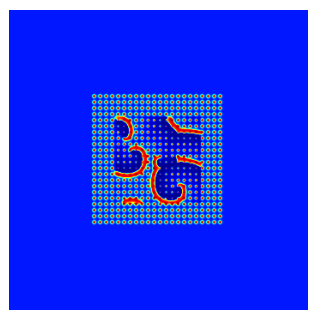

(b)

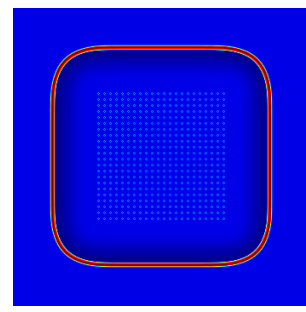

(d)

FIgURE 5: New waves generated outside the stimulated region in the SW suppression process. Tissue size : $350 \times 350$, stimulated region $150 \times 150$, spiral waves in the center $100 \times 100$. (a) $t=0 \mathrm{~ms}$; (b) $t=27.00 \mathrm{~ms}$; (c) $t=261.73 \mathrm{~ms}$; (d) $t=1123.80 \mathrm{~ms}$. $\left(k=1, A=83.53 \mu \mathrm{A} \mathrm{cm}{ }^{-2}, F=21.40 \mathrm{~Hz}, \theta=50 \%\right.$, SR $=1.96 \%$ in spiral waves region). 
1

3 electrodes grid with fixed frequency and duty cycle values and let us investigate

4 the influence of the variation of $k$ on the generation of waves. Fig. 6 shows the

5 minimum values of SR above which waves are generated ( $k=1$ and $k=0.75)$

6 versus the amplitude of $I_{\text {stim }}$. Compared to Fig. 2, when $k=1$, the threshold

7 allowing to generate waves is therefore much smaller than the necessary ampli-

s tude to suppress SW. It is thus impossible to eliminate SW without generating

9 new waves in this case. When $k=0.75$, the threshold of wave generation is

10 new ones.

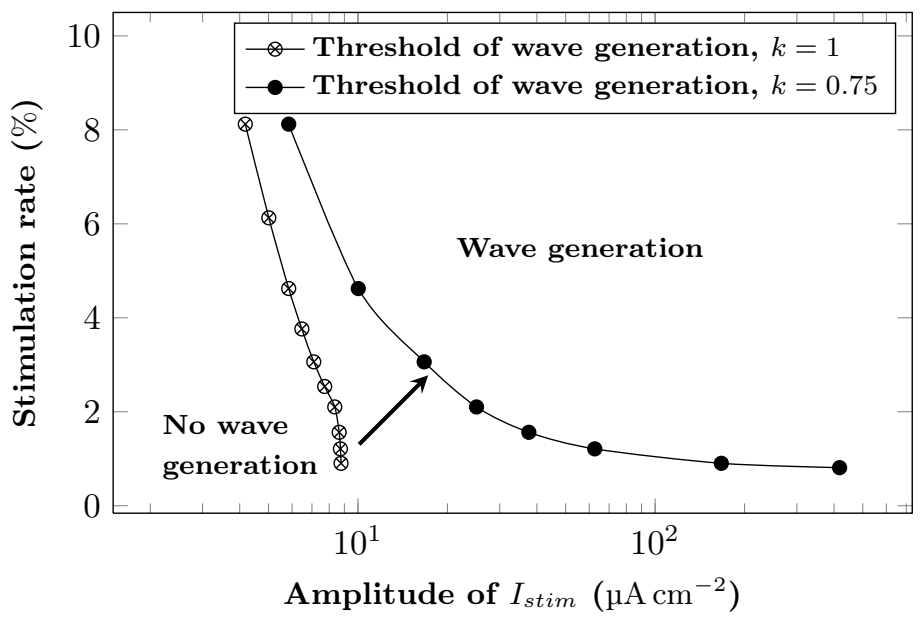

FiguRE 6: Comparison of thresholds of wave generation for $k=1$ and $k=0.75$. $(F=21.40 \mathrm{~Hz}, \theta=50 \%)$.

In order to investigate the influence of $k$ on wave generation, stimulations with a single electrode injected to the tissue initially in resting state have been performed. As shown in Fig. 7 , the minimum amplitude of $I_{\text {stim }}$ leading to wave generation can reach $A=2.09 \mathrm{~mA} \mathrm{~cm}^{-2}$ when $k=0.75$, which is 238 times larger than what is needed for $k=1\left(A=8.77 \mu \mathrm{Acm}^{-2}\right)$. So, in the next 
1 subsection, the effect of the modification of the parameter $k$ combined to the 2 SW suppression is studied.

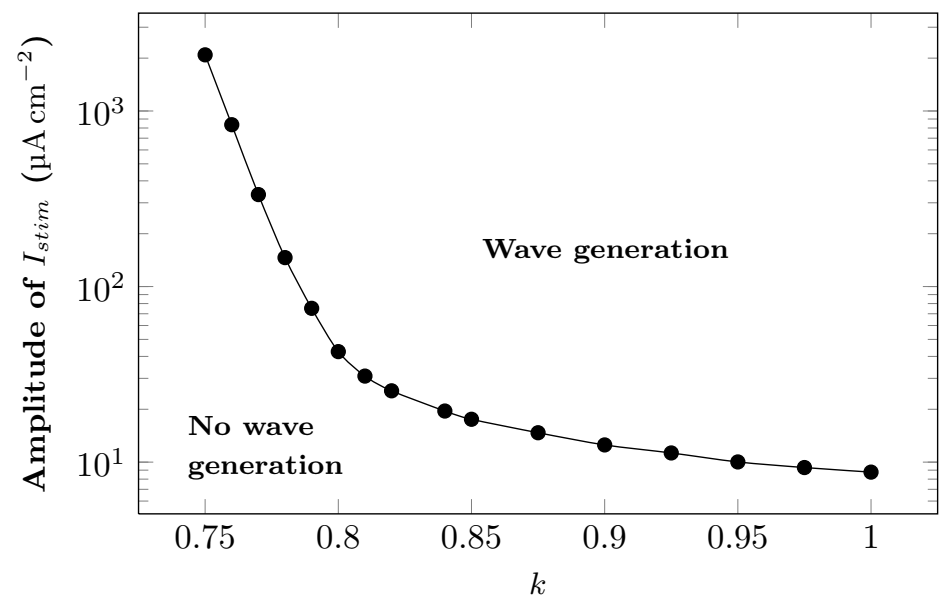

Figure 7: Amplitude of stimulus vs. Parameter $k$ characterizing the threshold of waves generation using one stimulating electrode. $(F=21.40 \mathrm{~Hz}, \theta=50 \%)$.

\section{3.3. Hybrid stimulation}

$4 \quad$ From precedent results, we know that suppressing locally SW by electrical

5 stimuli without generating new waves may be obtained by modifying the value

6 of the parameter $k$. Biologically, it could correspond to use pharmacological 7 agents in order to reduce the conductance of the sodium channels. This yields a

8 hybrid method. First, our simulation results show that when $k<0.73$, it is even

9 not possible to excite the tissue. The cells have only sub-threshold oscillations

10 even if the stimulation strength becomes substantial. Namely, considering the

11 fact that FHN model is a fast-slow dynamical system, the velocity of traveling

12 wave depends mainly on the fast dynamics i.e on the depolarization phase of the

13 action potential. In this case, the front velocity value is mainly a function of the

14 nonlinear threshold $\alpha$ of the sodium conductance and the diffusion parameter

${ }_{15} D[34,35]$ and depends also on the value of $k$. There exists a velocity threshold,

16 below which the system is unstable and waves fail to propagate through the

17 tissue [30]. Since $k$ controls the dynamics of sodium current, it is clear that a 
1 relatively small value of $k$ will result in a too low excitability of stimulated tissue 2 and the collapse of the waves.

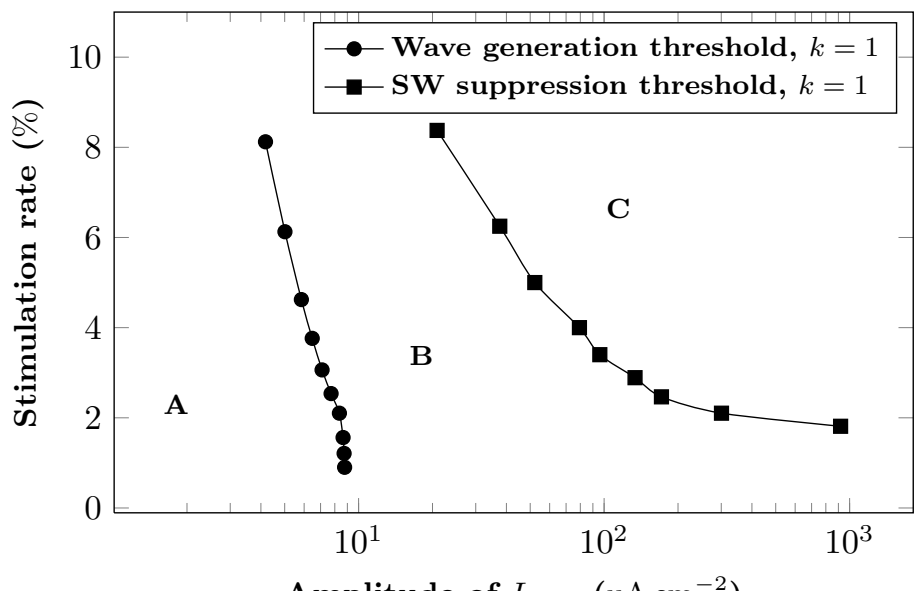

Amplitude of $I_{\text {stim }}\left(\mathrm{\mu A} \mathrm{cm}^{-2}\right)$

FiguRE 8: Comparison of the waves generation threshold and the SW suppression threshold with $F=21.40 \mathrm{~Hz}, \theta=50 \%$. $k=1$.

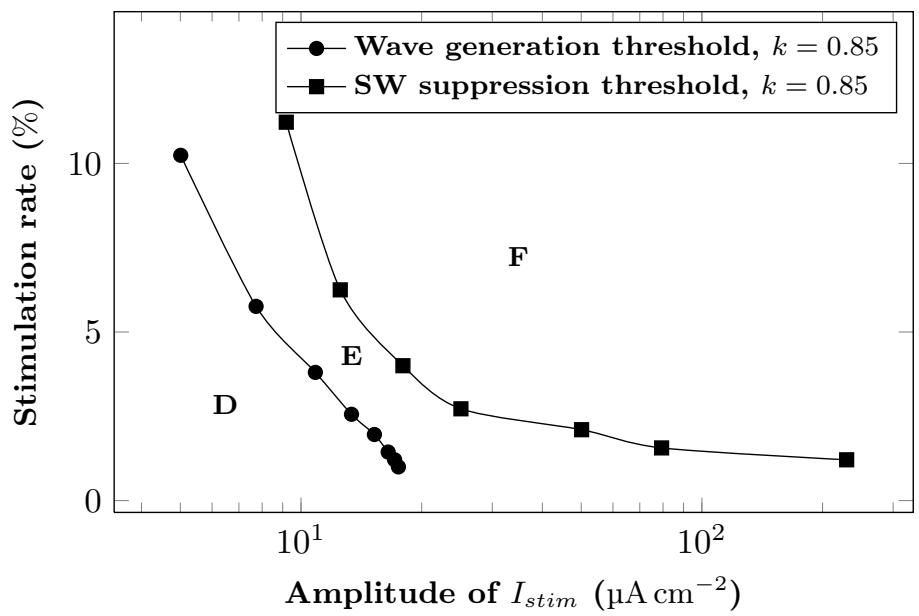

FiguRE 9: Comparison of the waves generation threshold and the SW suppression threshold with $F=21.40 \mathrm{~Hz}, \theta=50 \% . k=0.85$.

3 The results of the hybrid stimulation are shown in Figs. 8, 9, 10. As $k$ 4 decreases, two curves are getting nearer to each other : the wave generation 
1 threshold increases and the spiral wave suppression threshold decreases. When

$2 k=0.75$ (Fig. 10), they are totally reversed compared to those when $k=1$ (Fig.

3 8). It can be observed that in the regions $B$ and $E$, SW cannot be suppressed

4 and propagate as other new waves provoked by the stimulation in the tissue. The

5 suppression can be performed in $C, F$ and $I$ regions, but the stimuli generate

6 also new waves. Nothing happens (no suppression of SW and no generation of

7 waves) in $A, D$ and $G$, the stimulation process does not influence the dynamics

8 of the tissue. In the last region $H, \mathrm{SW}$ can be suppressed without yielding new

9 waves and the tissue is still excitable after the suppression of SW. This region is interesting in the optimization of the stimulation process.

\section{Conclusion and Discussion}

It is well known that atrial fibrillation (AF) begets AF [36]. However, according to Allessie's concept, AF can also self terminate if the spiral waves (SW) number is not enough to self maintain this cardiac arrhythmia [10]. In fact, the ideal atrial stimulation/defibrillation method should be able to suppress enough SW in a dedicated region to stop fibrillating for the whole heart, but without

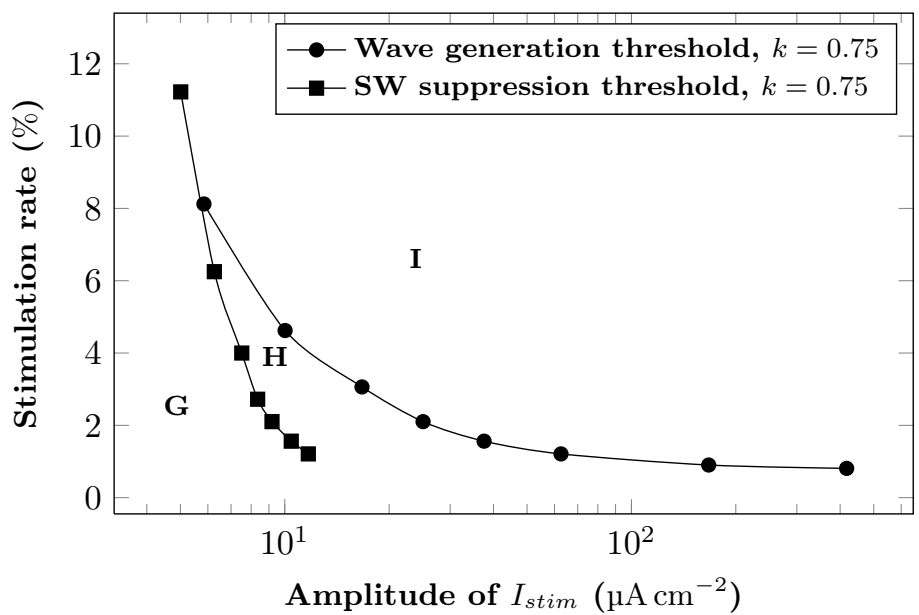

Figure 10: Comparison of the waves generation threshold and the SW suppression threshold with $F=21.40 \mathrm{~Hz}, \theta=50 \%$. $k=0.75$. 
1 generating new ones. In this intend, we studied here a simulated tissue of car-

2 diac cells using FHN model. Compared to other some realistic ionic models,

3 the FHN model is usually considered as a simple one and has its shortcomings.

4 However, we adopted it because it seems to be well adapted for modeling the

5 main dynamics of ion channels we needed in our study.

$6 \quad$ In this numerical model study, we showed that it may be possible to sup7 press spiral waves (SW) inside a local area by using a hybrid strategy. This

$\mathbf{8}$ strategy combined the optimization of multifocal stimulations delivered via a 9 grid of electrodes, and simulated sodium channel modification of the cardiac tissue. Although this strategy may only be a local and temporary solution (the confined area may be colonized by outside wandering SW), we believe that it could be of interest to restore the normal sinus rhythm under certain conditions considering for example Allessie's concept. In our study, we took into account physical parameters like the size and geometry of the electrode network as well as the number of the stimulating electrodes needed. We also identified some ideal pacing parameters in order to prevent from SW reinduction. However, in order to address this last issue, one of the options available is to modify the action potential dynamics (we choose here the sodium channel modification) in a simulated cardiac tissue.

In clinical practice, it is well known that antiarrhythmic drugs can optimize atrial defibrillation success rate. $\mathrm{Na}^{+}$channel blockers are commonly used to control the cardiac rhythm, and especially during AF [7, 37, 38, 39]. Whereas, their use can be limited by potentially serious side effects like other antiarrhythmic drugs, they remain at the first line therapy in AF treatment [40].

In summary, we showed that a hybrid strategy applied to the FitzHughNagumo model helped to identify specific regions where it was possible to suppress SW without provoking new reentries. In order to confirm these encouraging results, it would be of interest to apply the same strategy to more realistic ionic models. The next step could also be to use in vitro culture of cardiomyocytes $[41,42,43]$. The MEA (Microelectrode Array) technology could be a useful tool in testing the role of multifocal stimulation strategies on induced SW. As a 
1 clinical perspective, we may infer that resetting one or more local regions of the

2 left atrium via implanted array of electrodes connected to a dedicated pacing

3 device may help to eradicate enough SW to get back to normal sinus rhythm.

\section{References}

5 [1] K. Nanthakumar, J. Jalife, S. Massé, E. Downar, et al., Optical mapping of

$6 \quad$ langendorff-perfused human hearts : establishing a model for the study of

$7 \quad$ ventricular fibrillation in humans, American Journal of Physiology - Heart

$8 \quad$ and Circulatory Physiology 293 (1) (2007) H875-H880.

9 [2] H. J. J. Wellens, P. Brugada, J. Farre, Ventricular arrhythmias : Mecha-

10 nisms and actions of antiarrhythmic drugs, American Heart Journal 107 (5, Part 2) (1984) 1053-1057.

[3] J. Moreno, M. Warren, J. Jalife, Ionic currents and ventricular fibrillation dynamics, Revista Espanola de Cardiologia 57 (1) (2004) 69-79.

[4] R. D. White, Ventricular fibrillation and defibrillation : contemporary understanding of mechanisms, Anaesthesia, Pain, Intensive Care and Emergency A.P.I.C.E. (2007) 215-224.

[5] M. Mirowski, The automatic implantable cardioverter-defibrillator : An overview, Journal of the American College of Cardiology 6 (2) (1985) 461466.

[6] C. Patel, M. Salahuddin, A. Jones, A. Patel, G.-X. Yan, P. R. Kowey, Atrial fibrillation : Pharmacological therapy, Current Problems in Cardio$\log y 36(3)(2011) 87-120$.

[7] A. J. Camm, P. Kirchhof, G. Y. H. Lip, et al., Guidelines for the management of atrial fibrillation, European Heart Journal 31 (2010) 2369-2429.

[8] R. Weerasooriya, P. Khairy, J. Litalien, L. Macle, et al., Catheter ablation for atrial fibrillation : Are results maintained at 5 years of follow-up?, Journal of the American College of Cardiology 57 (2) (2011) 160-166. 
$1 \quad$ [9] J. Geller, S. Reek, C. Timmermans, others., Treatment of atrial fibrillation

2 with an implantable atrial defibrillator - long term results, European Heart

3 Journal 24 (23) (2003) 2083-2089.

4 [10] M. Allessie, W. Lammers, F. Bonke, J. Hollen, Experimental evaluation of 5 moe's multiple wavelet hypothesis of atrial fibrillation, Cardiac Electrophy6 $\quad$ siology and Arrhythmias. New York : Grune \& Stratton (1985) 265-276.

7 [11] J. Jalife, Rotors and spiral waves in atrial fibrillation, Journal of Cardio8 $\quad$ vascular Electrophysiology 14 (7) (2003) 776-780.

[12] F. H. Fenton, E. M. Cherry, H. M. Hastings, S. J. Evans, Multiple mechanisms of spiral wave breakup in a model of cardiac electrical activity, Chaos 12 (3) (2002) 852-892.

[13] V. Hakim, A. Karma, Spiral wave meander in excitable media: The large core limit, Phys. Rev. Lett. 79 (4) (1997) 665-668.

[14] H. Henry, Spiral wave drift in an electric field and scroll wave instabilities, Phys. Rev. E 70 (2) (2004) 026204-026208.

[15] V. Krinsky, V. Biktashev, A. Pertsov, Autowave approaches to cessation of reentrant arrhythmias, Ann. N. Y. Acad. Sci. 591 (Mathematical Approaches to Cardiac Arrhythmias) (2006) 232-246.

[16] J. Davidenko, R. Salomonsz, A. Pertsov, W. Baxter, J. Jalife, Effects of pacing on stationary reentrant activity : theoretical and experimental study, Circ. Res. 77 (6) (1995) 1166.

[17] K. Kamjoo, T. Uchida, T. Ikeda, M. Fishbein, A. Garfinkel, J. Weiss, H. Karagueuzian, P. Chen, Importance of location and timing of electrical stimuli in terminating sustained functional reentry in isolated swine ventricular tissues : evidence in support of a small reentrant circuit, Circulation 96 (6) (1997) 2048. 
[18] A. T. Stamp, G. V. Osipov, J. J. Collins, Suppressing arrhythmias in cardiac models using overdrive pacing and calcium channel blockers, Chaos 12 (3) (2002) 931-940.

[19] A. V. Panfilov, S. C. Müller, V. S. Zykov, J. P. Keener, Elimination of spiral waves in cardiac tissue by multiple electrical shocks, Phys. Rev. E 61 (4) (2000) 4644-4647.

[20] M. Allessie, C. Kirchhof, G. Scheffer, F. Chorro, J. Brugada, Regional control of atrial fibrillation by rapid pacing in conscious dogs, Circulation 84 (4) (1991) 1689-1697.

[21] S. Sridhar, S. Sinha, Controlling spatiotemporal chaos in excitable media using an array of control points, Europhys. Lett. 81 (2008) 50002.

[22] G. Osipov, B. Shulgin, J. Collins, Controlled movement and suppression of spiral waves in excitable media, Phys. Rev. E 58 (6) (1998) 6955-6958.

[23] H. Zhang, B. Hu, G. Hu, Suppression of spiral waves and spatiotemporal chaos by generating target waves in excitable media, Phys. Rev. E 68 (2) (2003) 26134.

[24] T. K. Shajahan, A. R. Nayak, R. Pandit, Spiral-wave turbulence and its control in the presence of inhomogeneities in four mathematical models of cardiac tissue, PLoS ONE 4 (3) (2009) 4738.

[25] C. Luo, Y. Rudy, A model of the ventricular cardiac action potential. depolarization, repolarization, and their interaction, Circ. Res. 68 (6) (1991) 1501.

[26] C. Luo, Y. Rudy, A dynamic model of the cardiac ventricular action potential. i. simulations of ionic currents and concentration changes, Circ. Res. 74 (6) (1994) 1071.

[27] K. Ten Tusscher, D. Noble, P. Noble, A. Panfilov, A model for human ventricular tissue, Am. J. Physiol. Heart Circ. Physiol. 286 (4) (2004) H1573. 
1

2

3

$4 \quad$ simulating nerve axon, Proceedings of the IRE 50 (10) (1962) 2061-2070.

5 [30] A. Scott, Neuroscience : a mathematical primer, Springer Verlag, 2002.

6 [31] B. Kogan, Introduction to Computational Cardiology, Springer-Verlag New

$7 \quad$ York Inc, 2009.

s [32] R. Clayton, A. Panfilov, A guide to modelling cardiac electrical activity - in anatomically detailed ventricles, Progress in Biophysics and Molecular

28] R. Fitzhugh, Impulses and physiological states in theoretical models of nerve membrane, Biophysical Journal 1 (6) (1961) 445-466.

29] J. Nagumo, S. Arimoto, S. Yoshizawa, An active pulse transmission line Biology 96 (1-3) (2008) 19-43.

[33] A. A. Dawodu, F. Monti, K. Iwashiro, M. Schiariti, R. Chiavarelli, P. E. Puddu, The shape of human atrial action potential accounts for different frequency-related changes in vitro, International Journal of Cardiology 54 (3) (1996) 237-249.

[34] S. Binczak, T. Sliwa, S. Jacquir, J. Bilbault, Reaction-diffusion network for geometric multiscale high speed image processing, Image and Vision Comput. 28 (6) (2010) 914-926.

[35] S. Binczak, J. M. Bilbault, Experimental propagation failure in a nonlinear electrical lattice, Int. J. Bifurcation Chaos 14 (2004) 1819-1830.

[36] M. C. Wijffels, C. J. Kirchhof, R. Dorland, M. A. Allessie, Atrial fibrillation begets atrial fibrillation : A study in awake chronically instrumented goats, Circulation 92 (7) (1995) 1954-1968.

[37] A. L. Goldin, Resurgence of sodium channel research, Annual Review of Physiology 63 (1) (2001) 871-894.

[38] J. Kneller, J. Kalifa, R. Zou, A. V. Zaitsev, M. Warren, O. Berenfeld, E. J. Vigmond, L. J. Leon, S. Nattel, J. Jalife, Mechanisms of atrial fibrillation 
1 termination by pure sodium channel blockade in an ionically-realistic ma-

$2 \quad$ thematical model, Circ Res 96 (5) (2005) 35-47.

3 [39] A. Burashnikov, J. M. Di Diego, A. C. Zygmunt, L. Belardinelli, C. Antzele-

$4 \quad$ vitch, Atrium-selective sodium channel block as a strategy for suppression

5 of atrial fibrillation : Differences in sodium channel inactivation between

6 atria and ventricles and the role of ranolazine, Circulation 116 (13) (2007)

$7 \quad 1449-1457$.

8 [40] C. Trial, Investigators. preliminary report : effect of encainide and flecai9 nide on mortality in a randomized trial of arrhythmia suppression after $10 \quad$ myocardial infarction, N Engl J Med 321 (6) (1989) 406-412.

11 [41] S. Jacquir, S. Binczak, D. Vandroux, G. Laurent, P. Athias, J. Bilbault, 12 Cardiac arrhythmias induced by an electrical stimulation at a cellular level, 13 IEEE Computers in Cardiology, 2008, Proceedings (2008) 625-628.

14 [42] S. Jacquir, G. Laurent, D. Vandroux, S. Binczak, J. Bilbault, P. Athias, 15 In vitro simulation of spiral waves in cardiomyocyte networks using multi16 electrode array technology, Archives of Cardiovascular Diseases, Elsevier $17 \quad 102(1)(2009)$ S63.

[43] S. Jacquir, S. Binczak, B. Xu, G. Laurent, D. Vandroux, P. Athias, J. Bilbault, Investigation of micro spiral waves at cellular level using a microelectrode array technology, Int. J. Bifurcation Chaos 21 (1) (2011) 209-223. 